\title{
HIV-Yield and Linkage in 13 Military Health Facilities in Cameroon: a Retrospective Study
}

\author{
Article by R. Medang ${ }^{1}$, U. Tamoufe ${ }^{2}$, G. Dion ${ }^{3}$, K. Saylors ${ }^{4}$, N. Ortiz ${ }^{5}$, M. Haile ${ }^{6}$, J.C. Aba ${ }^{7}$, A.

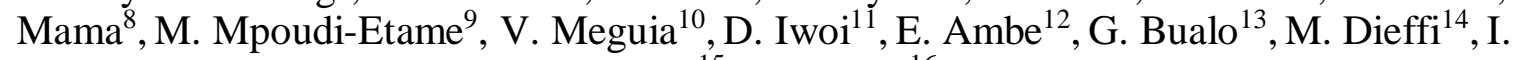 \\ Tonye $^{15}$, N. Manga ${ }^{16}$ \\ 1,2 Metabiota Cameroon \\ ${ }^{3}$ DoD/US Embassy Cameroon \\ 4, 5, ${ }^{6}$ Metabiota, Inc. Headquarters \\ 7, 10, 11,12, 13, 14, 15 Metabiota Cameroon \\ ${ }^{8,9,16}$ Cameroon Ministry of Defence \\ E-mail: rmedang@metabiota.com ${ }^{1}$,utamoufe@metabiota.com ${ }^{2}$,shirriad@yahoo.com ${ }^{3}$, \\ ksaylors@metabiota.com ${ }^{4}$,nortiz@metabiota.com ${ }^{5}$, mhaile@metabiota.com ${ }^{6}$, \\ aakam@metabiota.com ${ }^{7}$, mamnted@yahoo.fr ${ }^{8}$, mmpoudi@yahoo.com ${ }^{9}$, \\ vmeguia@metabiota.com ${ }^{10}$,diwoi@metabiota.com ${ }^{11}$,eambe@metabiota.com ${ }^{12}$, \\ gbualo@metabiota.com ${ }^{13}$, ndieffi@yahoo.fr ${ }^{14}$, itonye@metabiota.com ${ }^{15}$, maonest2@gmail.com ${ }^{16}$
}

\section{Introduction}

Since a number of years, many changes and a great evolution have occurred in the process of care and treatment as well as program management of HIV/AIDS control, worldwide. Many terms and paradigms have in fact emerged in the field of HIV/AIDS. New terms and paradigms such as the 90-90-90 target by 2020, the "test and treat", the linkage or the yield. As a matter of fact and according to the UNAIDS [11], "Ending the AIDS epidemic is more than a historic obligation to the 39 million people who have died of the disease. It also represents a momentous opportunity to lay the foundation for a healthier, more just and equitable world for future generations." This will undoubtedly be an enormous achievement for mankind as a whole, for what has been one of the greatest fears of the last 30 years. Thus, one of the biggest demands to date to achieve the elimination of the HIV epidemic is certainly the need to make the treatment universal, through the availability of ARVs.it should be admitted that, not only must treatment be made available, but it must also be ensured that all those who have been initiated into antiretroviral treatment remain faithful to it throughout their lives; hence the notions of linkage to treatment and retention to treatment. It should be remembered that a good follow-up of the antiretroviral treatment not only reduces the viral load in the individual undergoing treatment, resulting in a better quality of life and a longer life but also reduces the capacity of the infected person to transmit the HIV infection to others. Therefore, advantages obviously exist for both the infected individual and the entire community. In this respect, a powerful momentum initiated by the UNAIDS [11], is now building towards a new narrative on HIV treatment and a new, final, ambitious, but achievable target:

By 2020, 90\% of all people living with HIV will know their HIV status; - By 2020, $90 \%$ of all people with diagnosed HIV infection will receive sustained antiretroviral therapy; - By 2020, 90\% of all people receiving antiretroviral therapy will have viral suppression."

On the basis of the above mentioned elements and information, many countries, if not all countries around the world, have embarked on a frenetic race to achieve these three goals. Objectives which in fact where set for the bid of the elimination of the HIV epidemic around the world. These objectives have led, among other things, to the development of new strategies and new options, notably those of test and treat (test and start), linkage or yield. The main principle is to make sure that the maximum of persons infected in a country (at least 90\%), are tested, and maintained under ART. This is in a bid to ensure that their viral load becomes undetectable, and therefore, they become themselves unable to transmit the HIV infection to other people. This is why, in an unprecedented way, various AIDS control programs around the world are trying by every means to detect as many infected people as possible. Not only should infected people be tested, they should also be initiated into treatment and maintained for life under treatment. That is the challenge. 
In reality, we should talk about the challenges. Challenge to be able to detect at least $90 \%$ of infected persons, challenge to be able to link to treatment at least $90 \%$ of all positive screened persons to treatment and keep them under treatment. It is needless to recall that the goals of ART are to prolong life for persons living with HIV/AIDS, prevent opportunistic infections, reduce viral load, and reduce transmission of HIV to other people. As such, the concept of test and treat has become paramount. Concept of ensuring that all positive screened persons are immediately put on treatment. Beyond putting people who have been tested positive under treatment, it will be necessary to ensure that the people under treatment are kept under treatment for the rest of their lives. However, before the challenge of treatment, there is that of testing. The challenge of testing refers in fact to the need to find as many HIV positive people as possible. In this respect, we should consider another concept, that of the "yield". The "yield" which is defined as the absolute number of positives by site, a health facility for instance. The "yield" reflects the percentage of tests done with HIV positive results. The information reflects group of individuals seeking services at a specific site, whereas HIV prevalence reflects percentage of population with HIV positive status. The information reflects target population (entire population or representative sample), regardless of testing behavior. The "yield" represents in reality, the capacity of a site to find the potential HIV positive people in a community. To try to resolve the challenge related to search for positive HIV cases, several actions are being undertaken at the health facility level. Among these actions, we will mention, among other things, active research of HIV positive cases through the multiplication of entry points for testing, or the offer of testing service to any patient or non-patient present in the health facility (HIV testing initiated by the service provider. Other solutions include accentuating HIV testing of people with tuberculosis (with regard to TB / HIV coinfection) or testing from index cases. The testing of index cases is indeed the offer of testing service to people who have had sexual intercourse especially with people already infected. Despite all these options, however, the situation remains worrying, as the objectives are far from being achieved. It is therefore necessary to make an assessment of the situation allowing better appreciation of gaps and shortcomings.

It is from this perspective that for the present study, which is in fact a retrospective study carried out in 13 military health facilities, in which we have sought to:

- Determine the yield and linkage to treatment levels achieved by 13 military sites in the PEFAR network in Cameroon;

- Compare the level of yield achieved by these 13 sites with the level of HIV prevalence;

- Determine possible predictors for those who were successfully linked to treatment

\section{Method}

\section{Type of study}

We carried out a retrospective study on primary data collected at 13 health facilities, from October 2015 to September 2016.

\section{Data source, study population and period}

Data were extracted from a database containing demographic, HIV counseling and testing services from 13 military health facilities distributed in two (2) Military regions. The analysis was based on a cascade of persons tested for HIV (adults and children), those tested HIV+, as well as those linked to ARV treatment. The period of the study covered was from October 2015 to September 2016, for a total of 22,518 persons tested.

\section{Outcome variables}

For each individual, age, sex, and HIV status were collected, as well military region, facility, unit, profession (civilian or military). Concerning age in particular, we considered the following age-groups: < $1 ; 1-4 ; 5-9 ; 10-14 ; 15-19 ; 20-24 ; 25-49 ; 50+$. The main outcome indicators on which we worked were "yield" and linkage rate.

\section{Statistical analyses}

We used the $\mathrm{Z}$ score to estimate the statistical difference between the observed overall yield (\%) and the national HIV prevalence $(\%)$ within the age group 15-49 years, but also the statistical difference among 
regions (yield), as well as those in relation to sex (yield). Concerning linkage, comparison was made between results obtained and the level set by UNAIDS (90\%).

The $X^{2}$ test of adjustment was used to find out relationship between the HIV+ status of individuals and their demographic characteristics (age group, sex, general testing)

The proportion of cases referred to treatment (within one month and not linked) was assessed for each demographic characteristic and military regions in comparison with the second ninety (90\%) set by the UNAIDS.

In secondary analyses, multivariable logistic regression was used to examine the association between linkage to treatment and possible predictors such as military region, facility, unit, sex and profession. A two-tailed testing was used, with a P-value $<0.05$ considered as statically significant. Analyses were conducted using SPSS version 24.

\section{Results}

A total number of 22,518 individuals were recorded as to have been tested for HIV in 13 military health facilities, distributed in 2 military region ( 7 sites in the $1^{\text {st }}$ military region and 6 in the $2^{\text {nd }}$ Military region) from October 2015 to September 2016. The median age of persons tested was 28.0 years. Among those tested, 1,837 were found HIV positive for a yield of $8.2 \%$ (95\% CI: $7.8-8.5)$. Still on the overall testing, 1,163 individuals were successfully linked to treatment, for an overall linkage rate of $63.3 \%$ (95\% CI: 62.7 - 63.9). In terms of disaggregated data, 12.962 female were tested, among them 1,090 HIV positive, and 681 referred to treatment, providing a yield and linkage rate of respectively $8.4 \%$ (95\% CI: 7.9 - 8.9) and 62.5\% (95\% CI: 61.7 - 63.3), whereas 9,556 male were tested, among them 747 HIV positive, and 482 referred to treatment, providing a yield and linkage rate of respectively $7.8 \%$ (95\% CI: $7.3-8.3$ ) and 64.5\% (95\% CI: 63.5 - 65.5). In terms of testing in the two regions, 9.442 individuals were tested in the first military region, among them 863 HIV positive, and 789 referred to treatment, providing a yield and linkage rate of respectively $9.1 \%$ (95\% CI: $8.5-9.7$ ) and 91.4\% (95\% CI: 90.8 - 92.0), whereas 13,076 individuals were tested in the second military region, among them 974 HIV positive, and 374 linked to care, providing a yield and linkage rates of respectively $7.4 \%\left(\mathrm{CI}_{95 \%}\right.$ : 6.9 - 7.8) and $38.4 \%\left(\mathrm{CI}_{95 \%}\right.$ : 37.6 - 39.2). (See Table as well as Figure 1 and 2)

Concerning the age group 15-49 years which is the one considered by the 2011 DHS-MICS [10], the overall yield was $8.1 \%$ (95\% CI: $7.7-8.5$ ), whereas the National prevalence was $4.3 \%$. Still concerning the same age group, the yield was respectively $8.5 \%$ (95\% CI: 8.0 - 9.0) for female, and 7.8\% (95\% CI: 7.2 8.4) for male, whereas the National prevalence were respectively $5.6 \%$ and $2.9 \%$. Comparing observed HIV yield with HIV prevalence within 15-49 years in the following various groups (general population, female and male), and also comparing HIV yield in the 2 main Military regions, the results were as follow:

- Overall HIV yield in the age group $15-49$ years $(8.1 \%)$ was significantly different from the HIV prevalence $(4.3 \%)$ in the same age group $\left(X^{2}=651.8 ; \mathrm{p}<0.000001\right)$.

- In the same way, the yields in female age group 15 - 49 years (8.5\%) and in male age group 15-49 years $(7.6 \%)$ were significantly different from the HIV prevalence $(5.6 \%$ and $2.9 \%)$ in the same groups. $\left(X^{2}=174.0 \& 579.3\right.$ respectively; $\left.\mathrm{p}<0.000001\right)$.

When comparing the yield in the two Military regions, yield in the first Military region was $9.1 \%$, whereas yield of the second Military region was $7.4 \%$, resulting in a significant difference between the two proportions ( $\mathrm{z}$-score $=4.6 ; \mathrm{p}<0.00001)$.

Still in the same vein, comparison of the results in terms of linkage with the theoretical value set by the UNAIDS (90\% - second 90), showed a significant difference $(\mathrm{p}<0.0001)$ with the linkage levels of general and disaggregate sub populations (overall linkage: $63.3 \%$; linkage in second military region: $38.4 \%$; linkage in first military region: $91.4 \%$; linkage in Male: $64.5 \%$; linkage in female: $62.5 \%$ ). Considering linkage in male versus linkage in female, we had a $\mathrm{z}$-score $-3.1(\mathrm{p}=.002)$. Comparing the linkage rate of the $1^{\text {st }}$ Military Region versus $2^{\text {nd }}$ military Region, $\mathrm{z}$-score was 80.3 , p-value $<.0001$.

With multivariate analyses, facility, unit, sex, showed a 7\% (aOR .93, 95\% CI .85-1.02, $p=.162$ ), $28 \%$ (aOR: .72, 95\% CI .56-.91, $p=.008$ ), 32\% (aOR .68, 95\% CI .45-1.02, $p=.06$ ) decrease in linkage to treatment, On the other hand, it seemed like there was $31 \%$ and $67 \%$ increase in linkage to treatment concerning military region (aOR $1.31,95 \% \mathrm{CI} 0.83-2.06, p=.243$ ), as well as profession (aOR 1.67, $95 \%$ CI .79-3.54, $p=.175$ ). However, CI and p-values should be considered here. 


\section{Discussion}

From a total number of 22.518 individuals tested during 12 months (October 2015 - September 2016), our study considered a wide range of demographic and geographic characteristics, in which 13 health facilities were taken into account. The mean of persons tested by site was 1732.2 persons. The main outcomes within the context of test and treat (test and start) were the level of yield, and the level of linkage to care. Knowing that at the moment the focus is to identify as many HIV positive people as possible, to put them under treatment and to keep them under treatment. The ultimate objective being to reduce the viral load, for at least $90 \%$ of the people who were initiated under treatment. The study demonstrated a pretty high yield in general testing, female and male, in comparison with national prevalence in these various groups [10] of the newly tested HIV positive cases $(1,837), 40.7 \%$ were male, which was less than the $70.4 \%$ found in a study in China [9], and also less than the $74 \%$ in male, found. However, we can see that the yield among male in health facilities in this study, is far above the prevalence in male $(2.9 \%)$ given by the last DHS in Cameroon [10], at least for the age group 15-49. It should be recalled here that the 40.7\% (which is not the yield in males) is the percentage of HIV+ who are male among those found HIV+, and this takes into account male aged from $>1$ to $50+$. In fact, populations of all ages were considered here, and not only those sexually active. This also means that all types of HIV transmission were considered, from mother to child transmission, to blood transmission and sexual transmission. Nevertheless, the proportion of female tested HIV+ (59.3\%) among those found positive remains high in comparison with male, as it was also shown by the last DHS in Cameroon [10]. Even if, as can be seen, the levels of result seem different in health facilities, compared to general position. It may indeed be observed that within health facilities, the ratio of women / men of HIV + is 1.45 in health facilities, whereas it is 1.93 in general population. This again reflects a certain level of feminization of HIV infection in Cameroon.

Coming back to the concept of yield, it is worth mentioning that HIV yield is the absolute number of HIV positives detected within a health facility or a health unit. It allows programs to ensure that priority populations are reached, notably who complain of some symptoms which might be related to the HIV infection. In addition, make sure that those who are diagnosed are linked to care and treatment and benefit of viral load reduction. With an overall yield of $8.2 \%$, the program allowed 1,837 to know their HIV+ status, but among them, only $63.3 \%$ were initiated into ART, whereas the ultimate level of linkage to ART, should have been $90.0 \%$. Linkage to care and treatment is a crucial step towards viral load suppression and therefore towards HIV elimination.

Starting by children, the study demonstrated that the level of linkage to care for children $<1$ year, 1-4 years and 5-9 years, was respectively $20.0 \%, 94.7 \%$ and $116.7 \%$. In a study carried out by Adedimeli in Burundi, Rwanda and DRC [1], the proportion of children $\leq 24$ months of age at enrollment increased from $12.7 \%$ in $2004-05$ to $46.7 \%$ in $2012-13$, and from $9.6 \%$ in $2004-05$ to $24.2 \%$ in $2012-13$ for ART initiation. We can then observe here the level of linkage to care demonstrated by the military health facilities in Cameroon and in some age groups (1-4: 94.7\%; 5-9: 116.7\%) was far above the one described by the above mentioned study (24.2\%). In the United States of America (USA), a study on HIV Testing, Care Referral, and Linkage to Care by Philbin et al. [7], revealed that at 32 months, 2143 youth had been referred. Of these, 866 were linked to care through the Care Initiative within 42 days and thus eligible for study inclusion. Of the linked youth, $90.8 \%$ were ultimately engaged in care. Time from HIV testing to referral (eg., $\leq 7$ days versus $>365$ days) was associated with engagement [adjusted odds ratio $=2.91 ; 95 \%$ confidence interval (CI): 1.43 to 5.94] and shorter time to engagement (adjusted hazard ratio $=1.41 ; 95 \%$ CI: 1.11 to 1.79 ). In our study, the linkage rate was $70.0 \%$ and $88.8 \%$ respectively for age groups $10-14$ and 15-19.

In other demographic characteristics, we can observe that linkage to care was respectively $62.5 \%$ in female, $64.5 \%$ in male, $91.4 \%$ in the $1^{\text {st }}$ Military Region, $38.4 \%$ in the $2^{\text {nd }}$ Military Region, and $63.3 \%$ overall. Only the linkage rate in the $1^{\text {st }}$ Military Region (91.4\%) was higher than the theoretical value of $90 \%$ ( $\mathrm{p}<0.0001)$. All the other linkage rates were below the theoretical value of $90 \%$ ( $\mathrm{p}<.0001)$. Maman et al. [5] in a study carried out in Malawi showed that of the newly diagnosed, $47.5 \%$ were linked to care within 3 months, linkage to care was higher among older participants (40-59 vs. 15-29, adjusted hazard ratio [aHR] 3.39, 95\% CI 1.83-6.26), women (aHR 1.73, 95\% CI 1.12-2.67) and those eligible for ART (aHR 1.61, 95\% CI 1.03-2.52). Still in the context of HIV+ individuals being included into pre-ART before 
being linked to ART, a study implemented in South Africa by Haber et al. [3], used non-parametric survival analysis to estimate a longitudinal HIV care cascade for a large population of people with HIV residing in rural KwaZulu-Natal, In that study, and among living patients, only $45 \%$ were linked to care, $39 \%$ were eligible for ART, 35\% initiated ART, and 33\% had reached therapeutic response. Once more here, we can observe a low level of linkage to care, when we compare to our study (63.3\%) or to the current reference level (90\%). Still talking of poor linkage to care, a project implemented in Poland by Kowalska et al. [4], used Cox-proportional hazard models to identify factors associated with being linked to care and starting cART. The project stated that in total 232 persons were diagnosed HIV-positive and 144 (62.1\% 95\%CI: 55.5-68.3) persons were linked to care. There was no change over time in linkage to care $(\mathrm{p}=0.48)$, while time to starting cART decreased $(\mathrm{p}=0.02)$. Multivariate factors associated with a lower rate of linkage to care were hetero/bisexual sexual orientation, lower education, not having an HIV-positive partner and not using condoms in a stable relationship. Multivariate factors associated with starting cART were lower education, recent year of linked to care, and first HIV RNA and CD4 cell count. Since our study did not consider factors such as level of education, sexual orientation, HIV status of sexual partner (because not present in our registers), we were not able to assess association with these types of factors with linkage to care or to treatment. However, other factors were considered in our study (military region, facility, unit of care, sex, profession). In fact with our study, linkage to care in male (64.5\%) for instance, was slightly higher than linkage in female $(62.5 \%)-(\mathrm{p}=.002)$. in relation to the two military regions, the linkage rate of the $1^{\text {st }}$ military Region $(91.4 \%)$ was also higher than the one of the $2^{\text {nd }}$ military Region $(38.4 \%)-(\mathrm{p}=$ .00001).

In general, regarding our study and in the context of the text and treat (or test and start), initiation to treatment was done the same day. This was also the case for a qualitative study carried out by Buregveya et al. [2] in Uganda among HIV infected women. That study described that initiation of lifelong ART was done the same day the mother tested HIV positive. Several women felt the counselling was inadequate and had reservations about taking ART for life. The main motivation to initiate and adhere to ART was the desire to have an HIV-free baby. Adherence was a challenge, ranging from not taking the drugs at the right time, to completely missing doses and clinic appointments. Support from their male partners and peer family support groups enhanced good adherence. Fear to disclose HIV status to partners, drug related factors (side effects and the big size of the tablet), and HIV stigma were major barriers to ART initiation and adherence. Transition from antenatal care to HIV chronic care clinics was a challenge due to fear of stigma and discrimination. However in our own study, factors like facility, unit, sex, rather decrease percentage in linkage to treatment, while other like military regions and profession did not show a clear association with linkage to treatment. As we have already stated, we were not able to establish a clear association between location of testing (be it military region, health facility or unit of care), with linkage to treatment. This was not the case for some other studies, like the prospective mixed-method cohort study implemented by Sanga et al in Tanzania [8]. For this study, at the end of 6 months, 78\% of all respondents had linked into care, with differences across testing models. 84\% (CI 81\% to 87\%, $\mathrm{n}=512$ ) of individuals tested at facility-based site were linked to care compared to $69 \%$ (CI $65 \%$ to $74 \%, \mathrm{n}=281$ ) of individuals tested at mobile/outreach. The median time to linkage was 1 day (IQR: 1-7.5) for facility-based site and 6 days (IQR: 3-11) for mobile/outreach sites. Participants tested at facility-based site were 78\% more likely to link than those tested at mobile/outreach when other variables were controlled (AHR $=1.78 ; 95 \%$ CI 1.52 to 2.07). HIV status disclosure to family/relatives was significantly associated with linkage to care (AHR=2.64; 95\% CI 2.05 to 3.39). In this same option, a study carried out by Yhia et al. [12] in the USA (Philadelphia), demonstrated that diagnosis in inpatient settings, counseling and testing centers, and correctional facilities resulted in a $23 \%$ (AHR $0.77,95 \%$ confidence interval $0.64-0.92), 46 \%(0.56,0.42-$ $0.72)$, and $75 \%(0.25,0.18-0.35)$ decrease in the hazard of linkage to care compared to diagnosis in medical care clinics, respectively. Additionally, the hazard of not linking to care was greater for black patients (vs. white), persons with IDU as their HIV risk factor (vs. heterosexual), and those diagnosed in 2010 (vs. 2011). Secondary analyses examining factors associated with timely linkage to care, within 3 month of HIV diagnosis, yielded similar results.

In contrast to the two studies presented above, our study was carried out mainly in health facilities. Therefore, we did not have to compare different types of HIV testing locations, but rather characteristics of health facilities, or units of care in health facilities. Finally, we should also admit that other variables like 
quality of counseling, social status of the HIV+ individual, measures taken for linkage procedures by the health facility, were not taken into account in our study.

\section{Conclusion}

In the overall context of HIV elimination throughout the World, the three "90s" set up by UNAIDS, HIV yield, linkage to treatment and retention are the main pillars. As a retrospective study based on the collection of data from hospital registries, this study has demonstrated the importance of HIV testing within health facilities, notably with the concept of HIV yield, which allows to reach priority populations, and diagnose HIV as early as possible it has also demonstrated various implications related to linkage to care and treatment, such as the lack of achievement of the theoretical level of $90 \%$ linkage, both for the general population and for certain disaggregate categories (female; male, military region 2). With this, it appears that efforts still need to be made in terms of linking HIV + individuals under treatment as well as maintaining them there (retention). In fact, Despite success in case finding, efforts need to be made to improve active linkage, adherence counseling and follow-up of HIV-positive individuals in order to achieve UNAIDS objectives, perhaps focusing on female who are less likely to link to treatment. However, since our data were of secondary type, it was not possible to analyse some particular variables. In fact, the study only managed to analyse available variables (military regions, health facilities, units of care, sex, profession...). We would have wished to assess linkage to treatment with some other factors such as, quality of HIV counseling, measures taken by the program to enhance linkage to treatment, other characteristics of HIV+ patients such as level of education, sexual status, relationship with sexual partner, and so one and so forth. Another limitation was also the fact that the program has not yet been able to integrate the concept of unique identifier for HIV+ individuals. This could have enabled us to have more information on patients included in the study. 


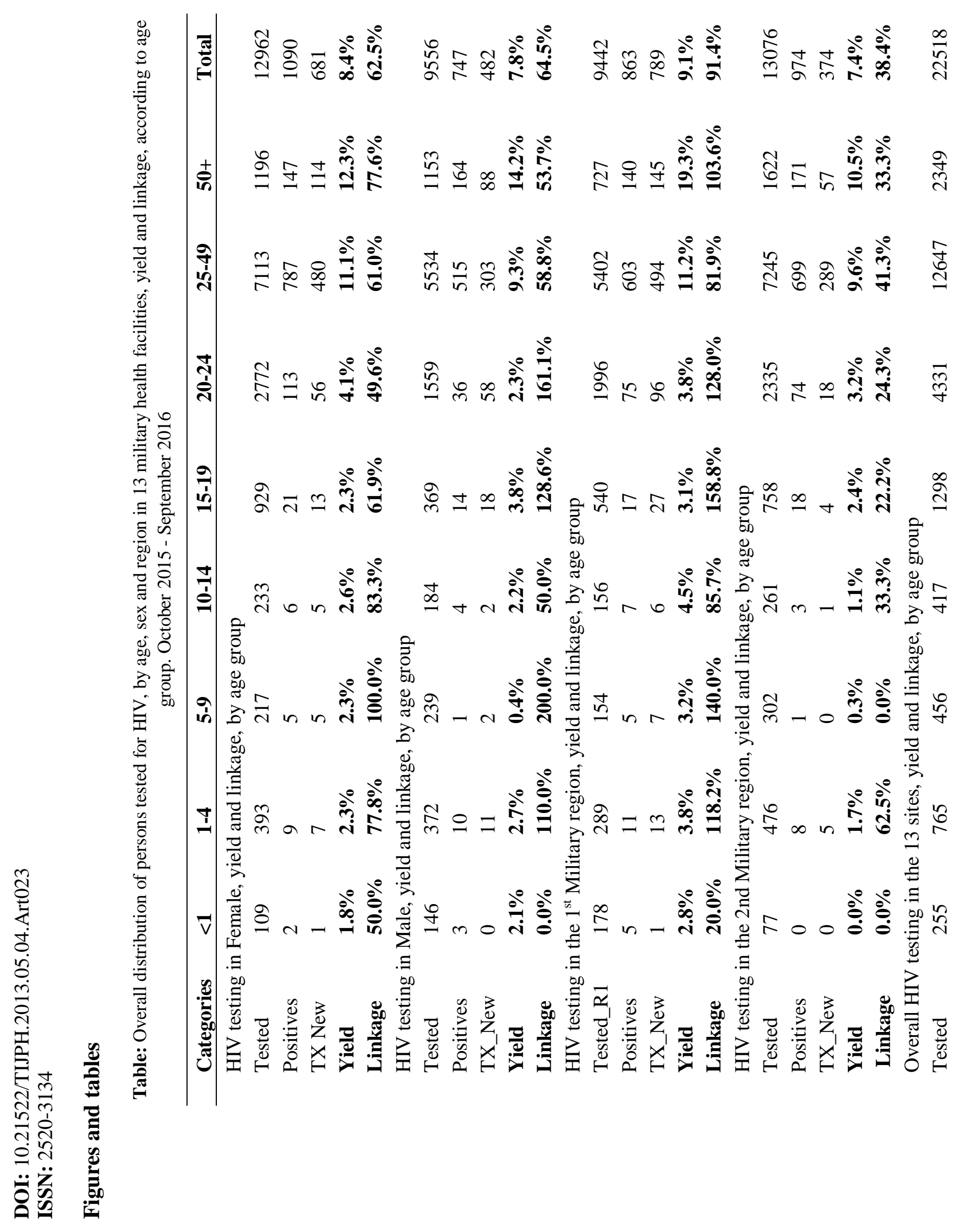




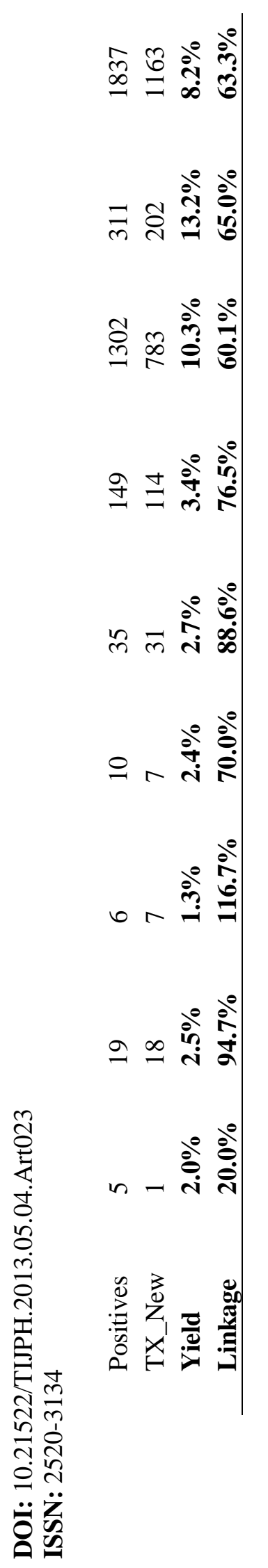


DOI: $10.21522 / \mathrm{TIJPH} .2013 .05 .04 . A r t 023$

ISSN: $2520-3134$

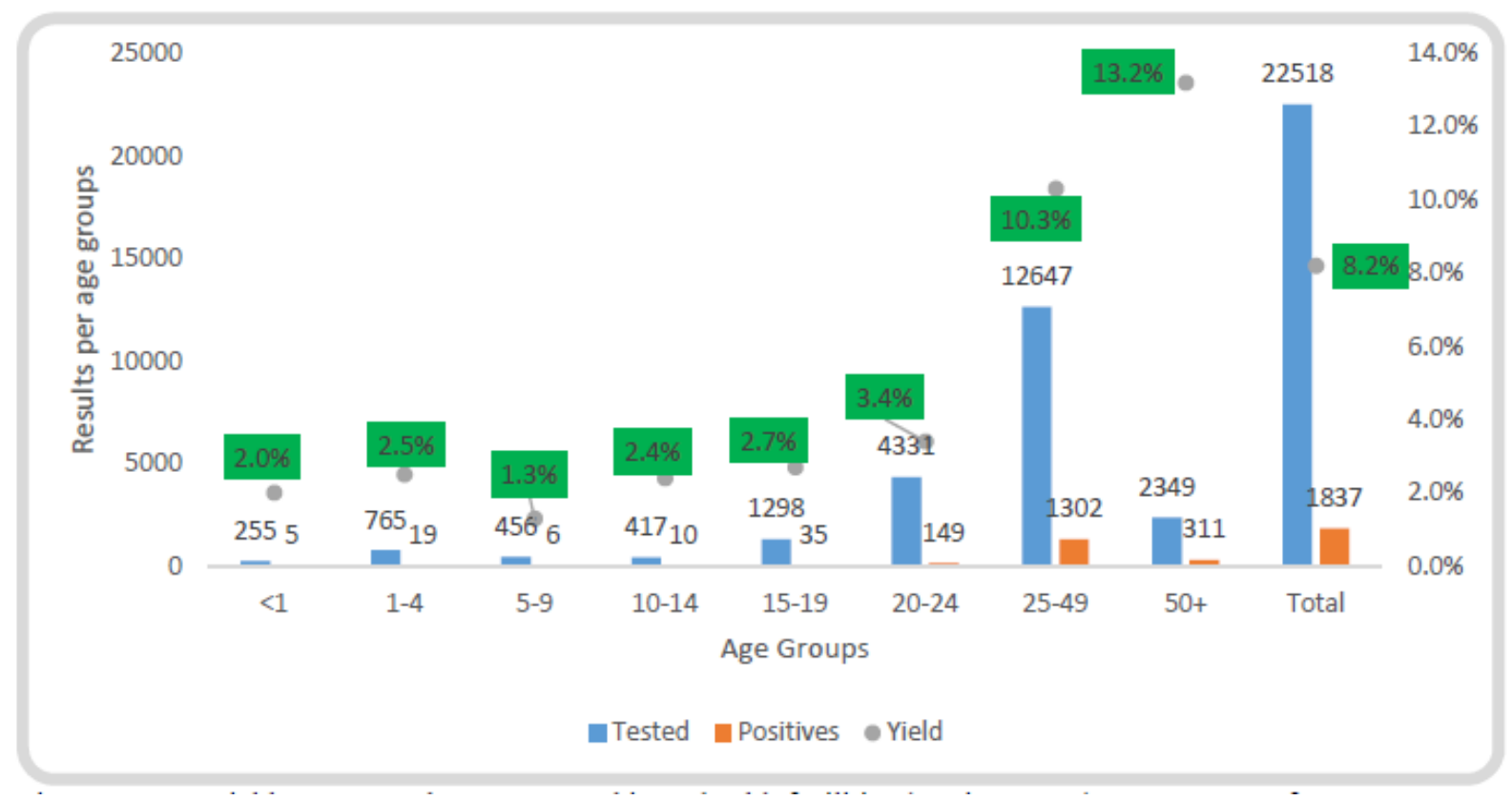

Figure 1. HIV Yield among total persons tested in 13 health facilities (region $1 \&$ 2) per age group from october 2015 to september 2016.

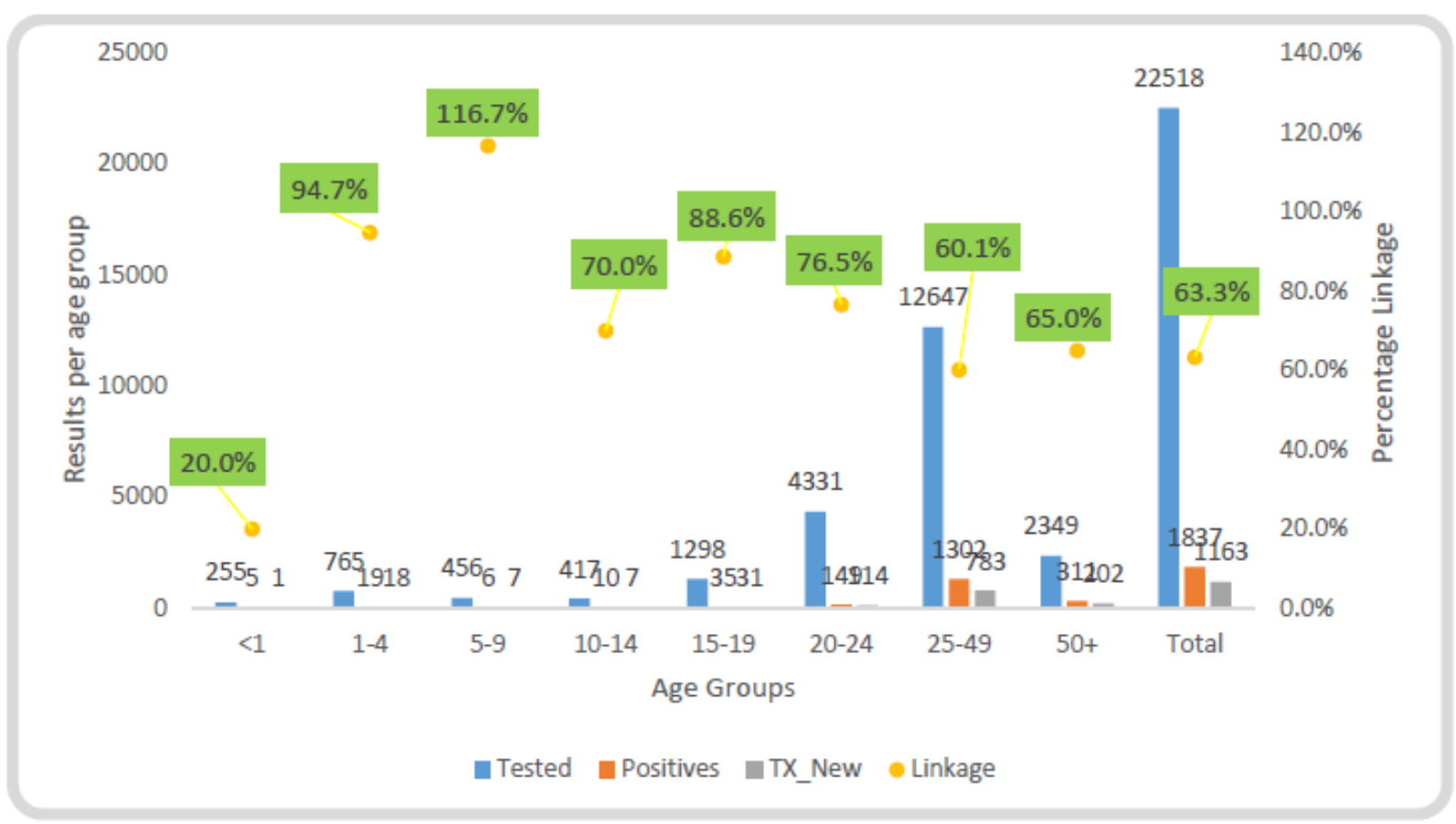

Figure 2. Linkage to care among total persons tested HIV+ in 13 health facilities (Region $1 \& 2$ ) per age group from October 2015 to September 2016. 


\section{References}

[1]. Adedimeji, A., Edmonds, A., Hoover, D., Shi, Q., Sinayobye, J. d'Amour, Nduwimana, M. ... Yotebieng, M. (2017). a at Enrollment into Care and at Antiretroviral Therapy Initiation in Central Africa. PLoS ONE, 12(1). https://doi.org/10.1371/journal.pone.0169871.

[2]. Buregyeya, E., Naigino, R., Mukose, A., Makumbi, F., Esiru, G., Arinaitwe, J. ... Wanyenze, R. K. (2017). Facilitators and barriers to uptake and adherence to lifelong antiretroviral therapy among HIV infected pregnant women in Uganda: a qualitative study. BMC Pregnancy and Childbirth, 17(1), 94. https://doi.org/10.1186/s12884017-1276-x.

[3]. Haber, N., Tanser, F., Bor, J., Naidu, K., Mutevedzi, T., Herbst, K. ... Bärnighausen, T. (2017). From HIV infection to therapeutic response: a population-based longitudinal HIV cascade-of-care study in KwaZulu-Natal, South Africa. The Lancet. HIV. https://doi.org/10.1016/S2352-3018(16)30224-7.

[4]. Kowalska, J. D., Shepherd, L., Ankiersztejn-Bartczak, M., Cybula, A., Czeszko-Paprocka, H., Firląg-Burkacka, E. ... Horban, A. (2016). Poor Linkage to Care despite Significant Improvement in Access to Early cART in Central Poland - Data from Test and Keep in Care (TAK) Project. PLoS ONE, 11(10). https://doi.org/10.1371/journal.pone.0162739.

[5]. Maman, D., Ben-Farhat, J., Chilima, B., Masiku, C., Salumu, L., Ford, N., .. Etard, J. F. (2016). Factors associated with HIV status awareness and Linkage to Care following home based testing in rural Malawi. Tropical Medicine \& International Health: TM \& IH, 21(11), 1442-1451. https://doi.org/10.1111/tmi.12772.

[6]. National Institute of Statistics (NIS) \& ICF Macro. (2011, février). Demographic and Health survey and Multiple Indicators Cluster Survey DHS-MICS Preliminary Report technical assistance of MEASURE DHS ICF International. [7]. Philbin, M. M., Tanner, A. E., DuVal, A., Ellen, J. M., Xu, J., Kapogiannis, B. ... Adolescent Trials Network for HIVAIDS Interventions. (2016a). HIV Testing, Care Referral, and Linkage to Care Intervals Affect Time to Engagement in Care for Newly Diagnosed HIV-Infected Adolescents in 15 Adolescent Medicine Clinics in the United States. Journal of Acquired Immune Deficiency Syndromes (1999), 72(2), $222-229$. https://doi.org/10.1097/QAI.0000000000000958.

[8]. Sanga, E. S., Lerebo, W., Mushi, A. K., Clowes, P., Olomi, W., Maboko, L., \& Zarowsky, C. (2017). Linkage into care among newly diagnosed HIV-positive individuals tested through outreach and facility-based HIV testing models in Mbeya, Tanzania: a prospective mixed-method cohort study. BMJ Open, 7(4), e013733. https://doi.org/10.1136/bmjopen-2016-013733.

[9]. Tang, H. L., Han, J., Li, J., Xu, J., \& Mao, Y. R. (2016). [Effects of standardized follow-up program among newly diagnosed HIV/AIDS cases in 2010]. Zhonghua Liu Xing Bing Xue Za Zhi = Zhonghua Liuxingbingxue Zazhi, 37(12), 1602-1607.

[10]. The 2011 DHSMICStradprelim_draft1 - DHSMICS_2011_preliminary_report.pdf. (s. d.). Consulté à l'adresse http://www.statistics-cameroon.org/downloads/EDS-MICS11/DHSMICS_2011_preliminary_report.pdf.

[11]. UNAIDS. (2014, octobre). 90-90-90: An ambitious treatment target to help end the AIDS epidemic.

[12].Yehia, B. R., Ketner, E., Momplaisir, F., Stephens, A., Dowshen, N., Eberhart, M. G., \& Brady, K. A. (2015). Location of HIV Diagnosis Impacts Linkage to Medical Care. Journal of acquired immune deficiency syndromes (1999), 68(3), 304-309. https://doi.org/10.1097/QAI.0000000000000459. 